\title{
Selenophen-2-yl-substituted thiocarbonyl ylides - at the borderline of dipolar and biradical reactivity
}

\author{
Mlostoń, Grzegorz ; Urbaniak, Katarzyna ; Linden, Anthony ; Heimgartner, Heinz
}

\begin{abstract}
The reactions of aryl (selenophen-2-yl) thioketones with CH2N2 occur with spontaneous elimination of $\mathrm{N} 2$, even at low temperature $\left(-65^{\circ}\right)$, to give regioselectively sterically crowded $4,4,5,5-$ tetrasubstituted 1,3-dithiolanes and/or a novel type of twelve-membered dithia-diselena heterocycles as dimers of the transient thiocarbonyl S-methanides. The ratio of these products depends on the type of substituent located at $\mathrm{C}(4)$ of the phenyl ring. Whereas the formation of the 1,3-dithiolanes corresponds to a $[3+2]$ cycloaddition of an intermediate thiocarbonyl ylide with the starting thioketone, the twelvememberd ring has to be formed via dimerization of the 'thiocarbonyl ylide' with an extended biradical structure.
\end{abstract}

DOI: https://doi.org/10.1002/hlca.201500050

Posted at the Zurich Open Repository and Archive, University of Zurich

ZORA URL: https://doi.org/10.5167/uzh-110376

Journal Article

Accepted Version

Originally published at:

Mlostoń, Grzegorz; Urbaniak, Katarzyna; Linden, Anthony; Heimgartner, Heinz (2015). Selenophen2-yl-substituted thiocarbonyl ylides - at the borderline of dipolar and biradical reactivity. Helvetica Chimica Acta, 98(4):453-461.

DOI: https://doi.org/10.1002/hlca.201500050 


\title{
Selenophen-2-yl Substituted Thiocarbonyl Ylides - at the Borderline of Dipolar and Diradical Reactivity
}

\author{
by Grzegorz Mlostoń*a ${ }^{\mathbf{a}}$ ), Katarzyna Urbaniak ${ }^{\mathbf{a}}$ ), Anthony Linden ${ }^{\mathbf{b}}$ ), and Heinz \\ Heimgartner*b)
}

a) University of Łódź, Department of Organic and Applied Chemistry, Tamka 12, PL91-403 Łódź (phone: +48 42 6355761; fax: +48 42 6655162; e-mail: gmloston@uni.lodz.pl)

b) University of Zürich, Department of Chemistry, Winterthurerstrasse 190, CH-8057 Zürich (phone:+41 44 6354282; fax: +41 44 6356812; e-mail: heinz.heimgartner@chem.uzh.ch)

Dedicated to Professor Christian Robl, Friedrich-Schiller-Universität Jena, on the occasion of his $60^{\text {th }}$ birthday 
The reactions of aryl (selenophen-2-yl) thioketones with diazomethane occur with spontaneous elimination of $\mathrm{N}_{2}$ even at low temperature $\left(-65^{\circ}\right)$, to give regioselectively sterically crowded 4,4,5,5-tetrasubstituted 1,3-dithiolanes and/or a novel type of 12-membered diselenadithia heterocycles as dimer of the transient thiocarbonyl S-methanides. The ratio of these products depends on the type of substituent located at $\mathrm{C}(4)$ of the $\mathrm{Ph}$ ring. Whereas the formation of the 1,3-dithiolanes corresponds with a [3+2] cycloaddition of an intermediate thiocarbonyl ylide with the starting thioketone, the 12-memberd ring has to be formed via dimerization of the 'thiocarbonyl ylide' with an extended biradical structure. 
1. Introduction. - The [3+2] cycloadditions belong to the most important reactions, which are of interest for both practical applications and development of reaction mechanism concepts. Especially important are synthetic methods applied for preparations of diverse heterocyclic products [1]. In addition to the classical series of reactive 1,3-dipolar species presented in the historical reviews by Rolf Huisgen [2], the S-centered 1,3-dipoles, such as thiocarbonyl S-imides [3a], thiocarbonyl S-oxides [3a], thiocarbonyl S-sulfides [3b], and especially thiocarbonyl ylides [3c], have been studied extensively in recent decades. One of the most characteristic features of $[3+2]$ cycloadditions is the concertedness of the creation of two $\sigma$-bonds in the course of the formation of five-membered heterocycles. However, in the case of the sterically crowded thiocarbonyl ylide $\mathbf{2 a}$, the reaction with electron-deficient alkenes $\mathbf{3}$, such as (E)-1,2-bis(trifluoromethyl)ethylene-1,2-dicarbonitrile or dimethyl dicyanofumarate $(\mathbf{3 b})$, follows a stepwise mechanism via a zwitterionic intermediate of type 4 [4][5] (Scheme 1).

\section{Scheme 1}

The stepwise mechanism was evidenced by the loss of stereospecifity, i.e., the formation of two stereoisomeric thiolanes of type $\mathbf{5}$ in the case of $\mathbf{3 b}$ [4], and the isolation of the seven-membered heterocycle 6 [5] or its derivatives obtained via its trapping with $\mathrm{MeOH}$ or $\mathrm{H}_{2} \mathrm{O}$ in the case of $\mathbf{3 a}$ [4][5]. The reactive thiocarbonyl ylide $\mathbf{2 a}$ can be generated conveniently via thermal cycloreversion of the 2,5-dihydro-1,3,4thiadiazole 1a [5], which is available by treatment of 2,2,4,4-tetramethyl-3thioxocyclobutan-1-one with diazomethane [6]. However in the case of aromatic thioketones, 1,3,4-thiadiazolines of type $\mathbf{1}$ are stable only below $-60^{\circ}$ and the corresponding thiocarbonyl ylides are generated in situ at ca. $-40^{\circ}$ [7]. 
An alternative mechanism for stepwise [3+2] cycloadditions was postulated by Raymond A. Firestone [8], and in that case, 1,3-diradicals were claimed to appear as reactive intermediates. However, the experimental evidence for this mechanism is vague, and to the best of our knowledge, the addition of a nitrone with substituted 1,3dienes, leading to a seven-membered heterocycle along with the expected fivemembered isoxazolidines, is the only example supporting the intermediacy of a biradical species [9]. In the case of thiocarbonyl ylides, intermediate diradicals were postulated for the formation of 1,3-dithiolanes based on computational studies [10]. In addition, the head-to-head dimerization of S-methanides derived from bisaryl thioketones, like thiobenzophenone S-methanide (2b), leading to 1,4-dithianes, could be explained via intermediate biradical species [11] (Scheme 2).

\section{Scheme 2}

2. Results and Discussion. - In our ongoing studies on reactions with aryl and hetaryl thioketones, we focused attention on the generation of S-methanides of type 7 derived from aryl (selenophen-2-yl) and bis(selenophen-2-yl) thioketones $\mathbf{8}$ (Scheme 3). Unexpectedly, the treatment of $\mathbf{8 a}\left(\mathrm{Ar}^{1}=\mathrm{Ph}\right)$ with diazomethane led to immediate evolution of $\mathrm{N}_{2}$, irrespective of the reaction temperature. The experiments performed at $20^{\circ}, 0^{\circ}$, and $-65^{\circ}$ afforded the same mixture of isomeric, sterically crowded $4,4,5,5-$ tetrasubstituted cis- and trans-1,3-dithiolanes 9a as major products. The structures of these compounds were elucidated on the basis of the ${ }^{13} \mathrm{C}-\mathrm{NMR}$ spectra, in which diagnostic signals of the $\mathrm{CH}_{2}$ groups were found at 30.3 and $31.0 \mathrm{ppm}$ [10b]. However, the ${ }^{1} \mathrm{H}-\mathrm{NMR}$ analysis of the mixture obtained with $8 \mathbf{a}$ showed that, along with isomeric dithiolanes 9a, another minor product with characteristic multiplets located at 2.63-2.69 
and 4.62-4.67 ppm was present. After separation of the mixture, this substance was obtained as slightly colored (beige) crystals. The ${ }^{13} \mathrm{C}-\mathrm{NMR}$ spectrum showed a set of 10 signals; two of them located at 29.3 and $60.6 \mathrm{ppm}$ were identified as $\mathrm{H}_{2} \mathrm{C}\left(\mathrm{sp}^{3}\right)$ and $\mathrm{HC}\left(\mathrm{sp}^{3}\right)$ groups, respectively. The ESI-HR-MS suggested the structure of a dimer of the intermediate thiocarbonyl ylide 7a. However, the spectroscopic data did not fit with the structure of the corresponding 1,4-dithiane, which could be expected as a product of typical head-to-head dimerization of bis-arylthiocarbonyl S-methanides [11] (Scheme 2).

\section{Scheme 3}

\section{Table 1}

Finally, the structure of the new dimer of the thiocarbonyl ylide $7 \mathbf{a}$ was established by X-ray crystallography as the hitherto unknown 12-membered macrocycle $10 a$.

\section{Figure}

It is worth mentioning that the analogous reaction with phenyl (thiophen-2-yl) thioketone led to a $c a$. 1:1 mixture of isomeric 1,3-dithiolanes of type 9 exclusively [13]. This observation suggests that the presence of a selenophen-2-yl substituent is a necessary condition for the formation of the dimer of type 10. In order to check the influence of substituents in the $\mathrm{Ph}$ ring of $\mathbf{8}$, a series of experiments with 4-substituted $\mathrm{Ph}$ analogs of $\mathbf{8 a}$ was performed (Table 1). The obtained results showed that electron withdrawing substituents in the starting thioketone favor the formation of the dimer $\mathbf{1 0 .}$ 
Remarkably, in the case of $\mathbf{8 e}$ containing the 4-nitrophenyl residue, the reaction performed at $-60^{\circ}$ occurred with vigorous evolution of $\mathrm{N}_{2}$, and the dimer $10 \mathrm{e}$ was obtained as the sole product according to ${ }^{1} \mathrm{H}-\mathrm{NMR}$ analysis. Similarly, the $4-\mathrm{CF}_{3}$ substituted thioketone $\mathbf{8 f}$ yielded under the same conditions the dimer 10f and only traces of the corresponding dithiolanes 9f (Table 1). On the other hand, the same reaction with $\mathbf{8 b}$ and $\mathbf{8 c}$ bearing electron-donating substituents gave predominantly mixtures of isomeric 1,3-dithiolanes $\mathbf{9 b}$ and $\mathbf{9 c}$, respectively, and only traces of the dimers 10 were detected. Finally, the reaction with the symmetric di(selenophen-2-yl) thioketone (8g) led to 1,3-dithiolanes as the major products [13], but traces of the unstable dimer $10 \mathrm{~g}$ could also be identified in the crude mixture.

These results indicate that the course of the observed [6+6] dimerization is favored in aryl (selenophen-2-yl) thiocarbonyl S-methanides 7 by the presence of electron-withdrawing substituents in the $\mathrm{Ph}$ ring. In our opinion, this type of dimerization of a thiocarbonyl ylide occurs via the intermediate delocalized diradical $\mathbf{1 1}$ (Scheme 4). Apparently, the dimerization is initiated by the formation of the $\mathrm{CH}_{2}-\mathrm{CH}_{2}$ bond and subsequent interaction of the radical centres at the $\alpha$-position of the selenophene rings. It seems likely that the reaction of diazomethane with hetaryl thioketones $\mathbf{8}$ under the applied conditions occurs without the formation of a corresponding 4,5-dihydro-1,3,4-thiadiazole of type 1, which may exist in the solution at very low temperature. The observed spontaneous elimination of $\mathrm{N}_{2}$ at low temperature differs significantly from reactions of diazomethane with diaryl and aryl hetaryl thioketones. 
3. Conclusions. - The present study shows that the in situ generated aryl (selenophen-2-yl) thiocarbonyl ylides $\mathbf{7}$ undergo either dimerization to give $\mathbf{1 0}$ or the competitive reaction with the starting thioketone $\mathbf{8}$, which yields 1,3 -dithiolanes $\mathbf{9}$ in a regioselective manner. The formation of $\mathbf{1 0}$ proves the intermediacy of a delocalized biradical 11. The yield of $\mathbf{1 0}$ depends on the substitution of the $\mathrm{Ph}$ ring in the starting thioketone. The competitive formation of 4,4,5,5-tetraaryl/hetaryl-substituted 1,3dithiolanes 9 can be proposed to occur also via diradical intermediates as suggested by computational studies [10a].

The authors thank the National Science Center (PL-Cracow) for financial support within the project Maestro (Grant Maestro-3; Dec-2012/06/A/ST5/00219).

\section{Experimental Part}

General. M.p.: MEL-TEMP. II (Aldrich); uncorrected. Column chromatography (CC): silica gel (70-230 mesh, Merck). IR Spectra: NEXUS FT-IR instrument; in KBr; absorptions in $\mathrm{cm}^{-1} .{ }^{1} \mathrm{H}-\mathrm{NMR}$ and ${ }^{13} \mathrm{C}-\mathrm{NMR}$ Spectra: BRUKER AVANCE III instrument $\left({ }^{1} \mathrm{H}\right.$ at 600 and ${ }^{13} \mathrm{C}$ at $\left.150 \mathrm{MHz}\right)$ using solvent signal as reference; in $\mathrm{CDCl}_{3}$; chemical shifts $(\delta)$ in ppm; coupling constants $J$ in $\mathrm{Hz}$. The majority of the ${ }^{13} \mathrm{C}$ signals were assigned with the aid of DEPT spectra. HR-ESI-MS were recorded on a Bruker maXis spectrometer in the Laboratory of Mass Spectrometry of the University of Zurich, on a Finnigan MAT 59 in the Laboratory of Mass Spectroscopy in CBMiM PAN Łódź, and on a Maldi SYNAPT G2-S HDMS in the Laboratory of the Institute of Organic Chemistry PAN Warsaw. Elemental analyses were performed in the Laboratory of the Faculty of Chemistry, University of Łódź. 
2. Starting Materials. All solvents and reagents are commercially available and were used as received. Thioketones $\mathbf{8}$ were obtained in a typical manner from the corresponding ketones [13] and Lawesson's reagent [14] in boiling toluene or benzene.

3. Reaction of Thioketones with Diazomethane. A soln. of $1 \mathrm{mmol}$ of the corresponding thioketone $\mathbf{8}$ in $1 \mathrm{ml}$ of dry THF was added drop-wise to a magnetically stirred soln. of $\mathrm{CH}_{2} \mathrm{~N}_{2}$ in $\mathrm{Et}_{2} \mathrm{O}$ at $-65^{\circ}$ according to a known procedure [11]. Only for the most reactive 4-nitrophenyl (selenophen-2-yl) thioketone (8f) was the reaction with $\mathrm{CH}_{2} \mathrm{~N}_{2}$ performed at $-85^{\circ}$. Under these conditions, decolorization of the thioketone soln. was observed immediately after addition of each portion. Pure products were isolated by CC or by crystallization.

3.1. Reaction with Phenyl (Selenophen-2-yl) Thioketone (8a). 4,5-Diphenyl-4,5di(selenophen-2-yl)-1,3-dithiolane (9a; 1:1 mixture of cis- and trans-isomer). Yield: $135 \mathrm{mg}$ (52\%). Beige crystals. M.p. $=162^{\circ}$ (decomp.; crystallization from hexane/ $\mathrm{CH}_{2} \mathrm{Cl}_{2}$ ). Purified by $\mathrm{CC}$ using a mixture of petroleum ether/ $\mathrm{CH}_{2} \mathrm{Cl}_{2}(3: 2)$ as the eluent. IR (KBr): 3050m, 2920m, 1628m, 1577m, 1487s, 1419m, 1282m, 1229s, 1187m, 1157m, 1031m, 792m, 752m, 696vs. ${ }^{1} \mathrm{H}-\mathrm{NMR}: 3.85,3.97\left(A B, J_{\mathrm{H}, \mathrm{H}}=9.2,2 \mathrm{H}, \mathrm{CH}_{2}\right.$ of cis-isomer); $4.02\left(s, 2 \mathrm{H}, \mathrm{CH}_{2}\right.$ of trans-isomer); $6.89\left(d, J_{\mathrm{H}, \mathrm{H}}=3.9,2 \mathrm{CH}_{\text {arom }}\right) ; 7.04(d d$, $\left.J_{\mathrm{H}, \mathrm{H}}=5.6,4.5,2 \mathrm{CH}_{\text {arom }}\right) ; 7.01-7.13\left(m, 6 \mathrm{CH}_{\text {arom }}\right) ; 7.19-7.22\left(m, 8 \mathrm{CH}_{\text {arom }}\right) ; 7.25(d$, $\left.J_{\mathrm{H}, \mathrm{H}}=7.2,2 \mathrm{CH}_{\mathrm{arom}}\right) ; 7.47\left(d, J_{\mathrm{H}, \mathrm{H}}=7.9,4 \mathrm{CH}_{\mathrm{arom}}\right) ; 7.49\left(d, J_{\mathrm{H}, \mathrm{H}}=7.9,4 \mathrm{CH}_{\text {arom }}\right) ; 7.91$ $\left(d, J_{\mathrm{H}, \mathrm{H}}=5.7,2 \mathrm{CH}_{\text {arom }}\right) ; 7.95\left(d, J_{\mathrm{H}, \mathrm{H}}=5.7,2 \mathrm{CH}_{\text {arom }}\right) .{ }^{13} \mathrm{C}-\mathrm{NMR}$ (mixture of cis- and trans-isomer): $30.3,31.0\left(2 \mathrm{CH}_{2}\right), 126.5,127.3,127.4,127.5,127.9,128.0,130.6$, $130.9,132.3,132.4,132.7,132.9\left(32 \mathrm{CH}_{\text {arom }}\right) ; 132.7,141.2,142.2,157.6\left(8 \mathrm{C}_{\mathrm{ar}}\right) . \mathrm{HR}-$ ESI-MS: $517.919210\left([M]^{+}, \mathrm{C}_{23} \mathrm{H}_{18} \mathrm{~S}_{2} \mathrm{Se}_{2}\right.$; calc. 517.918183).

(5Z,11Z)-6,11-Diphenyl-7,10-dithia-15,16-diselenatricyclo[10.2.1.1 $\left.1^{2,5}\right]$ hexadeca3,5,11,13-tetraene (10a). Yield $50 \mathrm{mg}$ (19\%) (partial decomposition during CC). Beige 
crystals. M.p. > $180^{\circ}$ (decomp., crystallization from hexane/ $\mathrm{CH}_{2} \mathrm{Cl}_{2}$ ). Isolated by $\mathrm{CC}$ using a mixture of petroleum ether/ $\mathrm{CH}_{2} \mathrm{Cl}_{2}$ (1:1) as the eluent. IR (KBr): $3026 s, 2951 s$, $1625 s, 1587 s, 1533 s, 1486 s, 1439 s, 1390 m, 1362 m, 1244 s, 1226 s, 1154 s, 1138 s, 1122 s$, 1075s, 1027s, 900s, 741vs, 730s, 709vs, 696vs. ${ }^{1} \mathrm{H}-\mathrm{NMR}: 2.63-2.69\left(m, 2 \mathrm{H}, \mathrm{CH}_{2} \mathrm{~S}\right)$; 4.62-4.67 ( $\left.m, 2 \mathrm{H}, \mathrm{CH}_{2} \mathrm{~S}\right) ; 4.82-4.83(m, 2 \mathrm{CHSe}) ; 6.21\left(d d, J_{\mathrm{H}, \mathrm{H}}=6.5,2.8,2 \mathrm{CH}=\right)$; $6.61\left(d d, J_{\mathrm{H}, \mathrm{H}}=6.5,1.0,2 \mathrm{CH}=\right) ; 7.29-7.31\left(m, 2 \mathrm{CH}_{\text {arom }}\right) ; 7.35-7.38\left(m, 4 \mathrm{CH}_{\text {arom }}\right)$; 7.47-7.50 ( $\left.m, 4 \mathrm{CH}_{\text {arom }}\right) .{ }^{13} \mathrm{C}-\mathrm{NMR}: 29.3\left(2 \mathrm{CH}_{2} \mathrm{~S}\right) ; 60.6$ (2 CHSe); 127.5, 128.2, 129.8, 134.5, $139.1\left(10 \mathrm{CH}_{\text {arom }}, 4 \mathrm{CH}=\right)$; 123.7, 141.7, $150.3\left(2 \mathrm{C}_{\text {arom }}, 2 \mathrm{C}=\mathrm{C}\right)$. HR-ESI-MS: $531.9344\left([M]^{+}, \mathrm{C}_{24} \mathrm{H}_{20} \mathrm{~S}_{2} \mathrm{Se}_{2}\right.$; calc. 531.9337).

3.2. Reaction with 4-Methoxyphenyl (Selenophen-2-yl) Thioketone (8b). 4,5Bis(4-methoxyphenyl)-4,5-di(selenophen-2-yl)-1,3-dithiolane (9b; 1:1 mixture of cisand trans-isomer). Yield: $205 \mathrm{mg}$ (71\%). Pale yellow crystals. M.p. > 183 (decomp.; crystallization from hexane/ $\mathrm{CH}_{2} \mathrm{Cl}_{2}$ ). Isolated after $\mathrm{CC}$ using a mixture of petroleum ether/ $\mathrm{CH}_{2} \mathrm{Cl}_{2}(3: 2)$ as the eluent. IR (KBr): $3054 m, 2927 m, 1604 m, 1577 m, 1507 \mathrm{vs}$, 1440m, 1294m, 1254vs, 1231m, 1184m, 1032m, 799m, 767m, 688s. ${ }^{1} \mathrm{H}-\mathrm{NMR}: 3.76$, $3.78(2 s, 6 \mathrm{H}, 2 \mathrm{MeO}) ; 3.85,3.95\left(A B, J_{\mathrm{H}, \mathrm{H}}=9.2,2 \mathrm{H}, \mathrm{CH}_{2}\right.$ of $c i s$-isomer $) ; 3.98(s, 2 \mathrm{H}$, $\mathrm{CH}_{2}$ of trans-isomer $) ; 6.62\left(d, J_{\mathrm{H}, \mathrm{H}}=8.7,2 \mathrm{CH}_{\text {arom }}\right) ; 6.69\left(d, J_{\mathrm{H}, \mathrm{H}}=8.8,4 \mathrm{CH}_{\text {arom }}\right) ; 6.92$ $\left(d, J_{\mathrm{H}, \mathrm{H}}=3.6,4 \mathrm{CH}_{\mathrm{arom}}\right) ; 7.01-7.03\left(m, 2 \mathrm{CH}_{\text {arom }}\right) ; 7.05-7.07\left(m, 2 \mathrm{CH}_{\mathrm{arom}}\right) ; 7.21\left(d, J_{\mathrm{H}, \mathrm{H}}\right.$ $\left.=3.6,2 \mathrm{CH}_{\text {arom }}\right) ; 7.31\left(d, J_{\mathrm{H}, \mathrm{H}}=5.2,4 \mathrm{CH}_{\text {arom }}\right) ; 7.34\left(d, J_{\mathrm{H}, \mathrm{H}}=8.8,4 \mathrm{CH}_{\text {arom }}\right) ; 7.88(d$, $\left.J_{\mathrm{H}, \mathrm{H}}=5.7,2 \mathrm{CH}_{\text {arom }}\right) ; 7.91\left(d, J_{\mathrm{H}, \mathrm{H}}=5.7,2 \mathrm{CH}_{\text {arom }}\right) .{ }^{13} \mathrm{C}-\mathrm{NMR}$ (mixture of cis- and transisomer): 30.1, $31.0\left(2 \mathrm{CH}_{2}\right)$; $55.2(4 \mathrm{MeO})$; 111.7, 112.6, 129.7, 128.0, 131.8, 131.9, $132.1,132.2,132.3,132.7\left(28 \mathrm{CH}_{\text {arom }}\right) ; 133.0,134.1,134.4,158.2,158.6,158.9$ (12 $\left.\mathrm{C}_{\mathrm{ar}}\right)$. HR-ESI-MS: $577.9413\left([M]^{+}, \mathrm{C}_{25} \mathrm{H}_{22} \mathrm{O}_{2} \mathrm{~S}_{2} \mathrm{Se}_{2}\right.$; calc. 577.9378).

(5Z,11Z)-6,11-Bis(4-methoxyphenyl)-7,10-dithia-15,16-diselenatricyclo[10.2.1.1 $1^{2,5}$ ]hexadeca-3,5,11,13-tetraene $(\mathbf{1 0 b})$ : detected in traces in the crude mixture; 
could not be isolated in pure form. ${ }^{1} \mathrm{H}-\mathrm{NMR}$ (selected signals): $2.62-2.67(m, 2 \mathrm{H}$, $\left.\mathrm{CH}_{2} \mathrm{~S}\right) ; 4.63-4.67\left(m, 2 \mathrm{H}, \mathrm{CH}_{2} \mathrm{~S}\right) ; 4.83-4.84$ ( $\left.m, 2 \mathrm{H}, 2 \mathrm{CHSe}\right)$.

3.3. Reaction with 4-Methylphenyl (Selenophen-2-yl) Thioketone (8c). 4,5-Bis(4methylphenyl)-4,5-di(selenophen-2-yl)-1,3-dithiolane (9c; 1:1 mixture of cis- and transisomer). Yield: $195 \mathrm{mg}$ (72\%). Pale yellow crystals. M.p. > 166 ${ }^{\circ}$ (decomp.; crystallization from hexane/ $\mathrm{CH}_{2} \mathrm{Cl}_{2}$ ). Isolated after $\mathrm{CC}$ by using a mixture of petroleum ether/ $\mathrm{CH}_{2} \mathrm{Cl}_{2}(3.5: 1.5)$ as the eluent. IR (KBr): $3024 m, 2915 m, 1626 m, 1508 \mathrm{~s}, 1441 m$, 1230s, 1193m, 1020m, 797m, 761m, 688vs. ${ }^{1} \mathrm{H}-\mathrm{NMR}: 2.38,2.40$ (2s, 6H, $\left.2 \mathrm{Me}\right) ; 3.81$, $3.93\left(A B, J_{\mathrm{H}, \mathrm{H}}=9.2,2 \mathrm{H}, \mathrm{CH}_{2}\right.$ of cis-isomer); 3.99 (s, 2H, $\mathrm{CH}_{2}$ of trans-isomer); 6.88 (d, $\left.J_{\mathrm{H}, \mathrm{H}}=3.7,2 \mathrm{CH}_{\text {arom }}\right) ; 6.90\left(d, J_{\mathrm{H}, \mathrm{H}}=8.1,4 \mathrm{CH}_{\text {arom }}\right) ; 6.97\left(d, J_{\mathrm{H}, \mathrm{H}}=8.1,4 \mathrm{CH}_{\text {arom }}\right) ; 7.01$ $\left(d d, J_{\mathrm{H}, \mathrm{H}}=5.6,3.9,2 \mathrm{CH}_{\text {arom }}\right) ; 7.05\left(d d, J_{\mathrm{H}, \mathrm{H}}=5.6,3.9,2 \mathrm{CH}_{\text {arom }}\right) ; 7.15\left(d, J_{\mathrm{H}, \mathrm{H}}=3.7,2\right.$ $\left.\mathrm{CH}_{\text {arom }}\right) ; 7.30\left(d, J_{\mathrm{H}, \mathrm{H}}=8.3,4 \mathrm{CH}_{\text {arom }}\right) ; 7.34\left(d, J_{\mathrm{H}, \mathrm{H}}=8.3,4 \mathrm{CH}_{\text {arom }}\right) ; 7.87\left(d, J_{\mathrm{H}, \mathrm{H}}=5.6\right.$, $\left.2 \mathrm{CH}_{\text {arom }}\right) ; 7.91\left(d, J_{\mathrm{H}, \mathrm{H}}=5.6,2 \mathrm{CH}_{\text {arom }}\right) .{ }^{13} \mathrm{C}-\mathrm{NMR}$ (mixture of cis- and trans-isomer): 20.9, $21.0\left(2 \mathrm{CH}_{2}\right) ; 30.1,30.9$ (4 Me); 127.2, 127.9, 128.0, 128.1, 130.5, 130.8, 131.8, 132.1, 132.3, 132.8 (28 $\left.\mathrm{CH}_{\text {arom }}\right)$; 136.9, 137.6, 138.0, 139.2, 158.3 (12 C $\left.\mathrm{C}_{\mathrm{ar}}\right)$. HR-ESIMS: $545.9515\left([M]^{+}, \mathrm{C}_{25} \mathrm{H}_{22} \mathrm{~S}_{2} \mathrm{Se}_{2}\right.$; calc. 545.9493). Anal. calc for $\mathrm{C}_{25} \mathrm{H}_{22} \mathrm{~S}_{2} \mathrm{Se}_{2}$ (544.49): C 55.15, H 4.07, S 11.78; found: C 55.36, H 4.48, S 11.71.

(5Z,11Z)-6,11-Bis(4-methylphenyl)-7,10-dithia-15,16-diselenatricyclo[10.2.1.1 $1^{2,5}$ hexadeca-3,5,11,13-tetraene (10c): traces, detected and identified spectroscopically in the crude mixture. ${ }^{1} \mathrm{H}-\mathrm{NMR}$ (selected signals): $2.63-2.68(\mathrm{~m}, 2 \mathrm{H}$, $\left.\mathrm{CH}_{2} \mathrm{~S}\right) ; 4.65-4.69$ ( $\left.m, 2 \mathrm{H}, \mathrm{CH}_{2} \mathrm{~S}\right) ; 4.85-4.88$ ( $\left.m, 2 \mathrm{H}, 2 \mathrm{CHSe}\right)$.

3.4. Reaction with 4-Chlorophenyl (Selenophen-2-yl) Thioketone (8d). 4,5-Bis(4chlorophenyl)-4,5-di(selenophen-2-yl)-1,3-dithiolane (9d; 1:1 mixture of cis- and transisomer): traces, detected and identified by ${ }^{1} \mathrm{H}-\mathrm{NMR}$ in the crude mixture. ${ }^{1} \mathrm{H}-\mathrm{NMR}$ : $3.85,3.99\left(A B, J_{\mathrm{H}, \mathrm{H}}=9.2,2 \mathrm{H}, \mathrm{CH}_{2}\right.$ of $c i s$-isomer $) ; 4.03\left(s, 2 \mathrm{H}, \mathrm{CH}_{2} \mathrm{~S}\right.$ of trans-isomer). 
(5Z,11Z)-6,11-Bis(4-chlorophenyl)-7,10-dithia-15,16-diselenatricyclo-

[10.2.1.1 $1^{2,5}$ hexadeca-3,5,11,13-tetraene (10d): Yield: $50 \mathrm{mg}(16 \%)$. Pale brown crystals. M.p. > $174^{\circ}$ (decomp.; crystallization from hexane/ $\mathrm{CH}_{2} \mathrm{Cl}_{2}$ ). Isolated after $\mathrm{CC}$ using a mixture of petroleum ether/ $\mathrm{CH}_{2} \mathrm{Cl}_{2}(1: 1)$ as the eluent; partially decomposed during chromatographic purification. IR (KBr): 3028m, 2954m, 1630m, 1565m, 1533s, 1482vs, 1419s, 1395s, 1341m, 1238s, 1222m, 1161s, 1140m, 1120m, 1087m, 1011s, 900s, 762vs, 722s, 674s. ${ }^{1} \mathrm{H}-\mathrm{NMR}: 2.61-2.67\left(m, 2 \mathrm{H}, \mathrm{CH}_{2} \mathrm{~S}\right)$; 4.57-4.62 (m, 2H, $\left.\mathrm{CH}_{2} \mathrm{~S}\right)$; 4.86-4.87 (m, 2H, 2 CHSe); $6.25\left(d d, J_{\mathrm{H}, \mathrm{H}}=6.6,2.7,2 \mathrm{CH}=\right) ; 6.56$ (br. $d, J_{\mathrm{H}, \mathrm{H}}=6.6,2$ $\mathrm{CH}=) ; 7.31,7.41\left(\mathrm{AB}, J_{\mathrm{H}, \mathrm{H}}=8.5,8 \mathrm{CH}_{\mathrm{arom}}\right) .{ }^{13} \mathrm{C}-\mathrm{NMR}: 29.2\left(2 \mathrm{CH}_{2} \mathrm{~S}\right) ; 60.6(2 \mathrm{CHSe})$; $128.4,131.1,134.2,139.7\left(8 \mathrm{CH}_{\text {arom }}, 4 \mathrm{CH}=\right)$; $122.3,133.4,140.0,150.8$ (4 $\mathrm{C}_{\text {arom }}, 2$ $\mathrm{C}=\mathrm{C})$. HR-ESI-MS: $599.8564\left([M]^{+}, \mathrm{C}_{24} \mathrm{H}_{18} \mathrm{Cl}_{2} \mathrm{~S}_{2} \mathrm{Se}_{2}\right.$; calc. 599.8557).

3.5. Reaction with 4-Nitrophenyl (Selenophen-2-yl) Thioketone (8e). (5Z,11Z)6,11-Bis(4-nitrophenyl)-7,10-dithia-15,16-diselenatricyclo[10.2.1.1 $\left.1^{2,5}\right]$ hexadeca-

3,5,11,13-tetraene (10e). Yield: $165 \mathrm{mg}$ (53\%). Yellow crystals. M.p. > 230 (decomp.; crystallization from hexane/ $\mathrm{CH}_{2} \mathrm{Cl}_{2}$ ). IR (KBr): $3058 m, 2954 m, 1628 m, 1587 m, 1527 m$, 1422m, 1301vs, 1237m, 1198m, 1108m, 1078m, 846s, 769s, 715m, 618m. ${ }^{1} \mathrm{H}-\mathrm{NMR}:$ 2.68-2.72 ( $\left.m, 2 \mathrm{H}, \mathrm{CH}_{2} \mathrm{~S}\right) ; 4.56-4.61\left(m, 2 \mathrm{H}, \mathrm{CH}_{2} \mathrm{~S}\right) ; 4.94-4.95(m, 2 \mathrm{H}, 2 \mathrm{CHSe}) ; 6.39$ $\left(d d, J_{\mathrm{H}, \mathrm{H}}=6.6,2.8,2 \mathrm{CH}=\right) ; 6.60\left(\right.$ br. $\left.d, J_{\mathrm{H}, \mathrm{H}}=6.6,2 \mathrm{CH}=\right) ; 7.65,8.23\left(A B, J_{\mathrm{H}, \mathrm{H}}=8.7,8\right.$ $\left.\mathrm{CH}_{\text {arom}}\right) .{ }^{13} \mathrm{C}-\mathrm{NMR}: 32.1\left(2 \mathrm{CH}_{2} \mathrm{~S}\right) ; 63.5$ (2 $\left.\mathrm{CHSe}\right) ; 123.7,129.9,131.0,139.0$ (8 $\mathrm{CH}_{\text {arom }} 4 \mathrm{CH}=$ ); 142.0, 143.1, 149.4, 149.9 (4 $\left.\mathrm{C}_{\text {arom }}, 2 \mathrm{C}=\mathrm{C}\right)$. HR-ESI-MS: 621.90329 $\left([M]^{+}, \mathrm{C}_{24} \mathrm{H}_{18} \mathrm{~N}_{2} \mathrm{O}_{4} \mathrm{~S}_{2} \mathrm{Se}_{2}\right.$; calc. 621.90395).

3.6. Reaction with 4-(Trifluoromethyl)phenyl (Selenophen-2-yl) Thioketone (8f). The cyclodimer (5Z,11Z)-6,11-Bis[4-(trifluoromethyl)phenyl]-7,10-dithia-15,16diselenatricyclo[10.2.1.1 $1^{2,5}$ hexadeca-3,5,11,13-tetraene (10f) was obtained only as a mixture with ketone $\mathrm{R}^{1} \mathrm{COR}^{2}\left(\mathrm{R}^{1}=4-\mathrm{CF}_{3} \mathrm{C}_{6} \mathrm{H}_{4}, \mathrm{R}^{2}=\right.$ selenophen-2-yl) and identified by 
NMR spectroscopy. All attempts to isolate $\mathbf{1 0 f}$ in a pure form, either chromatographically or by crystallization, were unsuccessful.

3.7. Reaction with Bis(selenophen-2-yl) Thioketone (8g). 4,4,5,5Tetra(selenophen-2-yl)-1,3-dithiolane (9g). Yield: $200 \mathrm{mg}$ (65\%; undergoes partial decomposition during chromatographic purification). Beige crystals. M.p. $>170^{\circ}$ (decomp.; crystallized from hexane/ $\mathrm{CH}_{2} \mathrm{Cl}_{2}$ ). Isolated by $\mathrm{CC}$ using a mixture of petroleum ether $/ \mathrm{CH}_{2} \mathrm{Cl}_{2}(3: 2)$ as the eluent. IR (KBr): $3087 m, 3053 m, 1631 m, 1437 m$, 1412m, 1328m, 1230s, 1128m, 1101m, 1056m, 1022m, 846m, 733m, 687vs. ${ }^{1} \mathrm{H}-\mathrm{NMR}:$ $4.13\left(s, \mathrm{CH}_{2}\right) ; 7.12\left(d d, J_{\mathrm{H}, \mathrm{H}}=5.8,4.0,4 \mathrm{CH}_{\text {arom }}\right) ; 7.21\left(d d, J_{\mathrm{H}, \mathrm{H}}=4.0,1.0,4 \mathrm{CH}_{\text {arom }}\right)$; $7.98\left(d d, J_{\mathrm{H}, \mathrm{H}}=5.8,1.0,4 \mathrm{CH}_{\text {arom }}\right) .{ }^{13} \mathrm{C}-\mathrm{NMR}: 30.9\left(\mathrm{CH}_{2}\right) ; 60.0\left(2 \mathrm{C}_{\mathrm{q}}\right) ; 128.2,132.6$, $133.8\left(12 \mathrm{CH}_{\text {arom }}\right) ; 152.5$ (4 $\left.\mathrm{C}_{\text {arom }}\right)$. HR-ESI-MS: $625.71826\left([M]^{+}, \mathrm{C}_{19} \mathrm{H}_{14} \mathrm{~S}_{2} \mathrm{Se}_{4}\right.$; calc. 625.720434).

(5Z,11Z)-6,11-Bis(selenophen-2-yl)-7,10-dithia-15,16-diselenatricyclo[10.2.1.1 $1^{2,5}$ hexadeca-3,5,11,13-tetraene $(\mathbf{1 0 g})$. Observed as a minor product in the crude reaction mixture $\left({ }^{1} \mathrm{H}-\mathrm{NMR}\right)$. This product completely decomposed during attempted purification by $\mathrm{CC}$ or crystallization. ${ }^{1} \mathrm{H}-\mathrm{NMR}$ (selected signals: 2.63-2.69 ( $\left.m, 2 \mathrm{H}, \mathrm{CH}_{2} \mathrm{~S}\right) ; 4.54-4.61\left(m, 2 \mathrm{H}, \mathrm{CH}_{2} \mathrm{~S}\right) ; 4.86-4.87$ ( $\left.m, 2 \mathrm{H}, 2 \mathrm{CHSe}\right)$.

4. X-Ray Crystal Structure Determination of 10a (Table 2 and Figure) $\left.{ }^{2}\right)$. A crystal of 10a suitable for a low-temperature X-ray structure determination was obtained from hexane/ $\mathrm{CH}_{2} \mathrm{Cl}_{2}$. All measurements were made on an Agilent

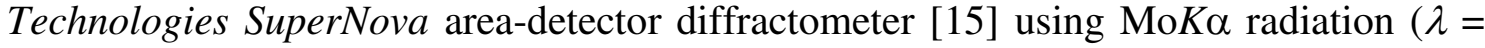

$\left.{ }^{2}\right)$ CCDC-1035301 contains the supplementary crystallographic data for this paper. These data can be obtained free of charge from the Cambridge Crystallographic Data Centre, via www.ccdc.cam.ac.uk/data_request/cif. 
$0.71073 \AA$ ) from a micro-focus X-ray source and an Oxford Instruments Cryojet XL cooler. Data reduction was performed with CrysAlisPro [15]. The intensities were corrected for Lorentz and polarization effects, and an empirical absorption correction using spherical harmonics [15] was applied. Equivalent reflections were merged. The data collection and refinement parameters are given in Table 2, and a view of the molecule is shown in the Figure. The structure was solved by direct methods using SHELXS-2013 [16], which revealed the positions of all non-H-atoms. The non-H-atoms were refined anisotropically. All of the $\mathrm{H}$-atoms were placed in geometrically calculated positions and refined by using a riding model where each $\mathrm{H}$-atom was assigned a fixed isotropic displacement parameter with a value equal to $1.2 \mathrm{U}_{\mathrm{eq}}$ of its parent atom. The refinement of the structure was carried out on $F^{2}$ by using full-matrix least-squares procedures, which minimized the function $\Sigma w\left(F_{\mathrm{o}}{ }^{2}-F_{\mathrm{c}}{ }^{2}\right)^{2}$. A correction for secondary extinction was not applied. There are two voids of $174 \AA^{3}$ per unit cell, which join together into channels running parallel to [001]. The SQUEEZE routine [17] of the PLATON program [18] indicated approximately 10 e per cavity, which would correspond with a $\mathrm{H}_{2} \mathrm{O}$ molecule. However, neither inclusion of a $\mathrm{H}_{2} \mathrm{O}$ molecule, even with partial occupancy, nor refinement with the data generated by SQUEEZE yielded any improvement in the results, so the original reflection data and the solvent-free model were retained for the final refinements. The maximum residual electron density peak is only $0.55 \mathrm{e}^{-3}$, so if solvent is present in the channels, it is diffuse. Neutral atom scattering factors for non-H-atoms were taken from Maslen, Fox and $O^{\prime} K e e f e$ [19a], and the scattering factors for H-atoms were taken from Stewart, Davidson and Simpson [20] Anomalous dispersion effects were included in $F_{\mathrm{c}}$ [21]; the values for $f^{\prime}$ and $f^{\prime \prime}$ were those of Creagh and McAuley [19b]. The values of the mass attenuation coefficients are 
those of Creagh and Hubbel [19c]. The SHELXL-2013 program [16] was used for all calculations.

Table 2. Crystallographic Data for Compound 10a

\section{REFERENCES}

[1] A. Padwa, W. H. Pearson, Eds., Synthetic Applications of 1,3-Dipolar Cycloaddition Chemistry Toward Heterocycles and Natural Products, J. Wiley \& Sons, New York, 2002.

[2] a) R. Huisgen, Angew. Chem. Int. Ed. Engl. 1963, 2, 565; b) R. Huisgen, in 1,3Dipolar Cycloaddition Chemistry, A. Padwa, Ed., J. Wiley \& Sons, New York, 1984, Vol. 1, pp. 1-176.

[3] a) G. Mlostoń, H. Heimgartner, Science of Synthesis, Update 2014, pp. 393-401;

b) G. Mlostoń, H. Heimgartner, Science of Synthesis, Update 2014, pp. 403-411;

c) G. Mlostoń, H. Heimgartner, Science of Synthesis, Update 2014, pp. 377-384.

[4] a) R. Huisgen, G. Mlostoń, H. Giera, E. Langhals, Tetrahedron 2002, 58, 507; b)

G. Mlostoń, R. Huisgen, H. Giera, Tetrahedron 2002, 58, 4185.

[5] R. Huisgen, G. Mlostoń, E. Langhals, T. Oshima, Helv. Chim. Acta 2002, 85, 2668.

[6] C. E. Diebert, J. Org. Chem. 1970, 35, 1501.

[7] G. Mlostoń, H. Heimgartner, Pol. J. Chem. 2000, 74, 1503; G. Mlostoń, H. Heimgartner, in '1,3-Dipolar Cycloaddition Chemistry Towards Heterocycles and Natural Products', Eds. A. Padwa, W. H. Pearson, J. Wiley \& Sons, New York, 2002, p. 315. 
[8] a) R. A. Firestone, J. Org. Chem. 1968, 33, 2285; b) R. A. Firestone, Tetrahedron 1977, 33, 3009.

[9] J. Baran, H. Meyer, J. Org. Chem. 1989, 54, 5774.

[10] a) R. Sustmann, W. Sicking, R. Huisgen, Chem. Eur. J. 2003, 9, 2245; b) R. Huisgen, G. Mlostoń, K. Polborn, R. Sustmann, Chem. Eur. J. 2003, 9, 2256.

[11] R. Huisgen, I. Kalvinsch, X. Li, G. Mlostoń, Eur. J. Org. Chem. 2000, 1685.

[12] C. K. Johnson, ORTEPII, Report ORNL-5138, Oak Ridge National Laboratory, Oak Ridge, Tennessee, 1976.

[13] G. Mlostoń, K. Urbaniak, K. Gębicki, P. Grzelak, H. Heimgartner, Heteroatom Chem. 2014, 25, 548 .

[14] M. Jesberger, T. P. Davis, L. Barner, Synthesis 2003, 1929.

[15] CrysAlisPro, Version 1.171.37.31d, Agilent Technologies, Yarnton, Oxfordshire, England, 2014.

[16] G. M. Sheldrick, Acta Crystallogr. Sect. A 2008, 64, 112.

[17] P. van der Sluis, A. L. Spek, Acta Crystallogr. Sect. A, 1990, 46, 194.

[18] A. L. Spek, Acta Crystallogr. Sect. D, 2009, 65, 148.

[19] a) E. N. Maslen, A. G. Fox, M. A. O'Keefe, in 'International Tables for Crystallography', Ed. A. J. C. Wilson, Kluwer Academic Publishers, Dordrecht, 1992, Vol. C, Table 6.1.1.1, pp. 477-486; b) D. C. Creagh, W. J. McAuley, in 'International Tables for Crystallography', Ed. A. J. C. Wilson, Kluwer Academic Publishers, Dordrecht, 1992, Vol. C, Table 4.2.6.8, pp. 219-222; c) D. C. Creagh, J. H. Hubbell, in 'International Tables for Crystallography', Ed. A. J. C. Wilson, Kluwer Academic Publishers, Dordrecht, 1992, Vol. C, Table 4.2.4.3, pp. 200206.

[20] R. F. Stewart, E. R. Davidson, W. T. Simpson, J. Chem. Phys. 1965, 42, 3175. 
[21] J. A. Ibers, W. C. Hamilton, Acta Crystallogr. 1964, 17, 781. 
Table 1. Reactions of Aryl (Selenophen-2-yl) Thioketones $\mathbf{8}$ with Diazomethane in THF $a t-65^{\circ}$

\begin{tabular}{|c|c|c|c|c|}
\hline Entry & $\mathrm{Ar}^{1}$ & $\mathbf{8}$ & $\left.\mathbf{9}\left(\text { Yield }^{\mathrm{a}}\right)^{\mathrm{b}}\right)$ & $\left.\mathbf{1 0}(\text { Yield })^{\mathrm{a}}\right)$ \\
\hline 1 & $\mathrm{Ph}$ & $\mathbf{a}$ & $\mathbf{a}(52 \%)$ & $\mathbf{a}(19 \%)$ \\
\hline 2 & $4-\mathrm{MeOC}_{6} \mathrm{H}_{4}$ & $\mathbf{b}$ & $\mathbf{b}(71 \%)$ & $\left.\mathbf{b}(\text { traces })^{\mathrm{c}}\right)$ \\
\hline 3 & $4-\mathrm{MeC}_{6} \mathrm{H}_{4}$ & $\mathbf{c}$ & $\mathbf{c}(72 \%)$ & $\left.\mathbf{c}(\text { traces })^{\mathrm{c}}\right)$ \\
\hline 4 & $4-\mathrm{ClC}_{6} \mathrm{H}_{4}$ & $\mathbf{d}$ & $\left.\mathbf{d}(\text { traces })^{\mathrm{c}}\right)$ & $\mathbf{d}(16 \%)$ \\
\hline 5 & $4-\mathrm{O}_{2} \mathrm{NC}_{6} \mathrm{H}_{4}$ & $\left.\mathbf{e}^{\mathrm{d}}\right)$ & - & $\mathbf{e}(53 \%)$ \\
\hline 6 & $4-\mathrm{F}_{3} \mathrm{CC}_{6} \mathrm{H}_{4}$ & $\mathbf{f}$ & $\left.\mathbf{f}^{\mathrm{c}}\right)$ & $\left.\mathbf{f}^{\mathrm{c}}\right)$ \\
\hline 7 & Selenophen-2-yl & $\mathbf{g}$ & $\mathbf{g}(65 \%)$ & $\left.\mathbf{g}(\text { minor })^{\mathrm{c}}\right)$ \\
\hline
\end{tabular}

a) Yield of isolated product

b) Ca. 1:1-mixtures of cis- and trans-isomer

c) Not isolated; detected by ${ }^{1} \mathrm{H}-\mathrm{NMR}$ in the crude mixture

d) Reaction carried out at $-85^{\circ}$ 
Table 2. Crystallographic Data for Compound 10a

Crystallized from

Empirical formula

Formula weight $\left[\mathrm{g} \mathrm{mol}^{-1}\right]$

Crystal color, habit

Crystal dimensions [mm]

Temperature $[\mathrm{K}]$

Crystal system

Space group

Z

Reflections for cell determination

$2 \theta$ range for cell determination $\left[{ }^{\circ}\right]$

Unit cell parameters $a[\AA]$

$b[\AA]$

$c[\AA]$

$\beta\left[^{\circ}\right]$

$V\left[\AA^{3}\right]$

$D_{x}\left[\mathrm{~g} \mathrm{~cm}^{-3}\right]$

$\mu(\operatorname{Mo~} K \alpha)\left[\mathrm{mm}^{-1}\right]$

Scan type

$2 \theta_{(\max )}\left[{ }^{\circ}\right]$

Transmission factors (min; max)

Total reflections measured

Symmetry independent reflections

Reflections with $I>2 \sigma(I)$

Reflections used in refinement

Parameters refined

Final $R(F)[I>2 \sigma(I)$ reflections $]$

$$
w R\left(F^{2}\right) \text { (all data) }
$$

Weights:

Goodness of fit

Final $\Delta_{\max } / \sigma$

$\Delta \rho(\max ; \min )\left[\mathrm{e} \AA^{-3}\right]$ hexane $/ \mathrm{CH}_{2} \mathrm{Cl}_{2}$

$\mathrm{C}_{24} \mathrm{H}_{20} \mathrm{~S}_{2} \mathrm{Se}_{2}$

530.34

yellow, prism

$0.10 \times 0.10 \times 0.23$

160(1)

monoclinic

$C 2 / c$

8

12146

5-61

26.2131(5)

$14.4025(3)$

12.0823(2)

99.6333(18)

4497.17(15)

1.566

3.480

$\omega$

60.7

$0.711 ; 1.000$

28288

6258

5019

6258

253

0.0265

0.0628

$w=\left[\sigma^{2}\left(F_{\mathrm{o}}{ }^{2}\right)+(0.027 P)^{2}+2.909 P\right]^{-1}$ where $P=\left(F_{\mathrm{o}}{ }^{2}+2 F_{\mathrm{c}}{ }^{2}\right) / 3$

1.042

0.002

$0.51 ;-0.33$ 
Legends

Scheme 1. Stepwise Reaction of a Sterically Crowded Thiocarbonyl Ylide 2a with Electron Deficient 1,2-Bis(trifluoromethyl)ethylene 1,2-Dicarbonitrile (3a)

Scheme 2. Generation of Thiobenzophenone S-Methanide (2b) and its Behavior: (a) in the Presence of Thiobenzophenone; (b) in the Absence of a Trapping Agent

Scheme 3. Se-Containing Thiocarbonyl Ylides Disclose the Nature of the Diradical Species

Scheme 4. Delocalized Diradical Intermediate

Figure. ORTEP plot [12] of the molecular structure of $\mathbf{1 0 a}$ (50\% probability ellipsoids; arbitrary numbering of the atoms). 
Scheme 1

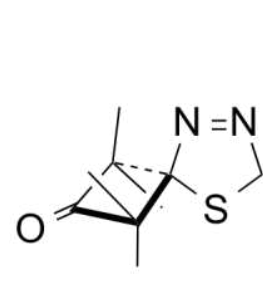

$1 a$

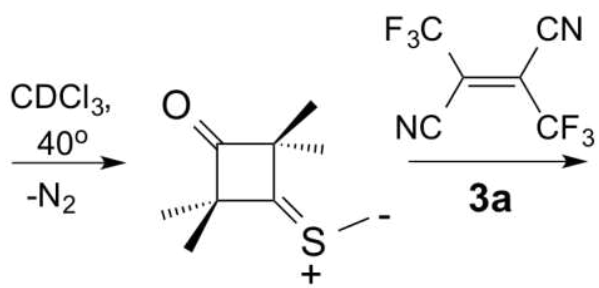

2a

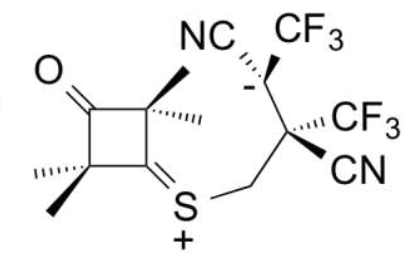

4
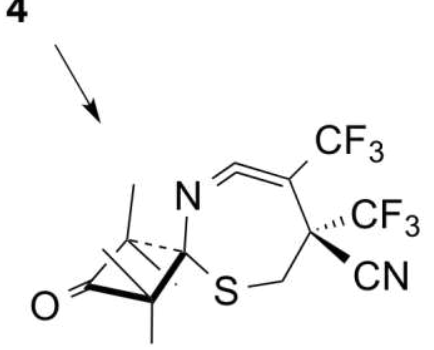

6

Scheme 2

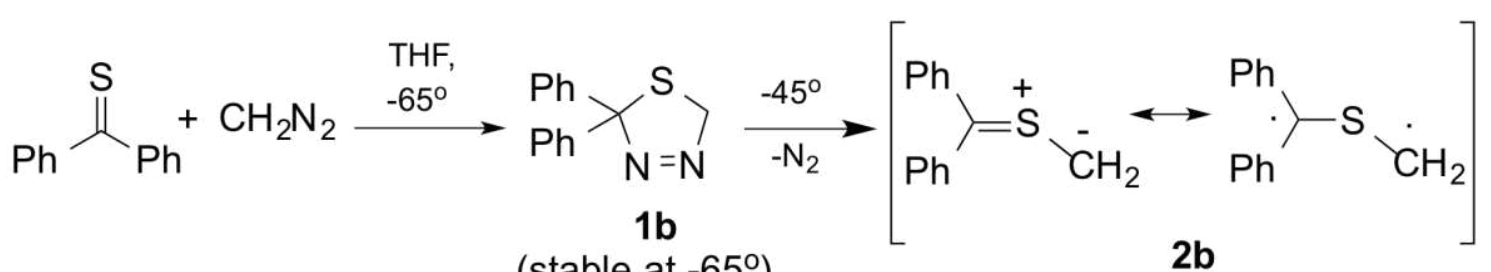
(stable at $-65^{\circ}$ )

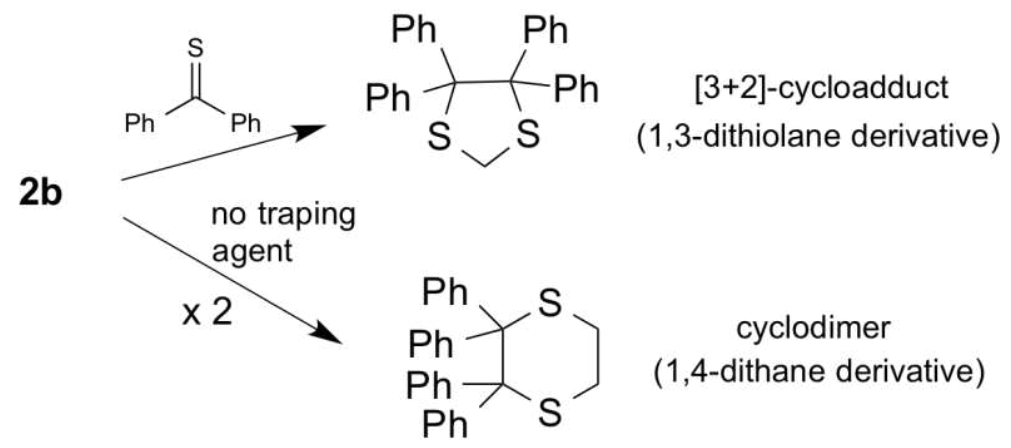


Scheme 3
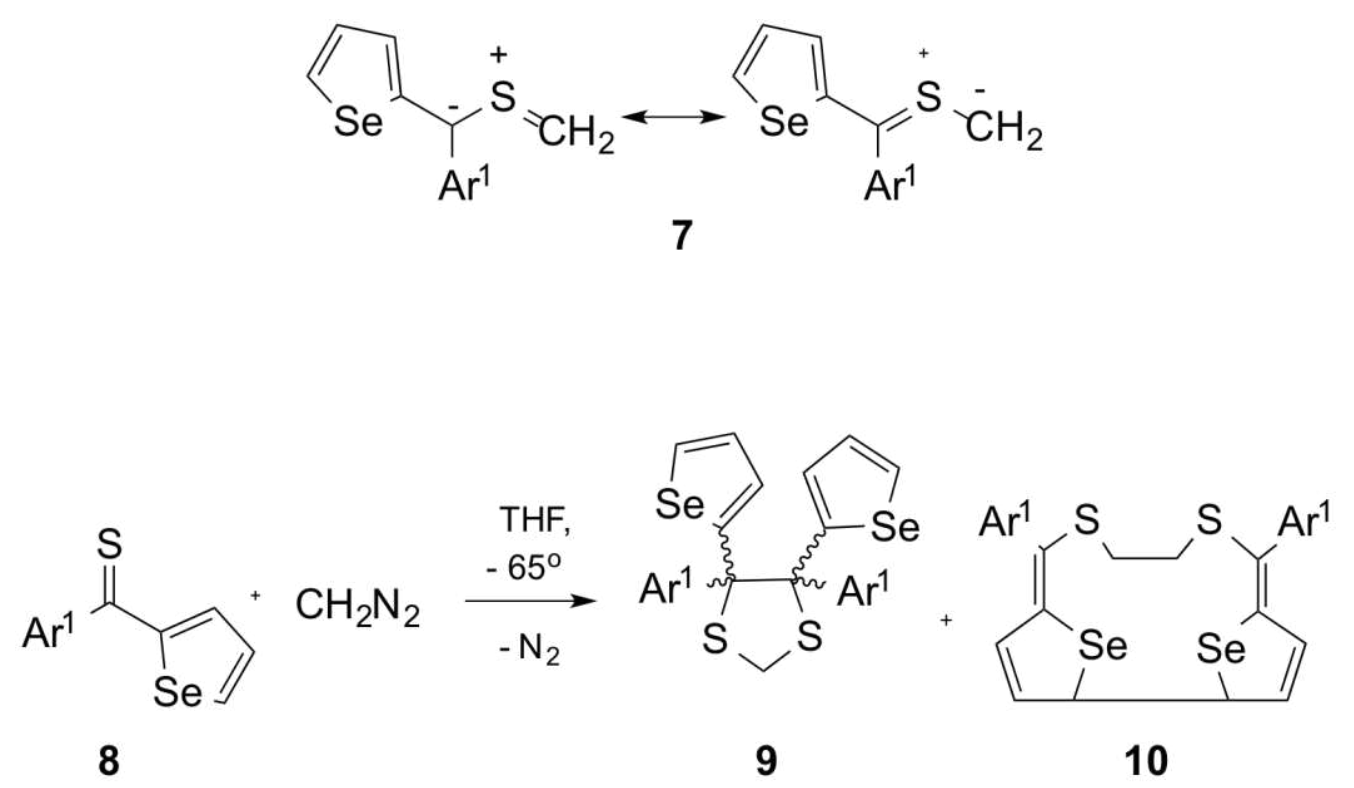

Scheme 4

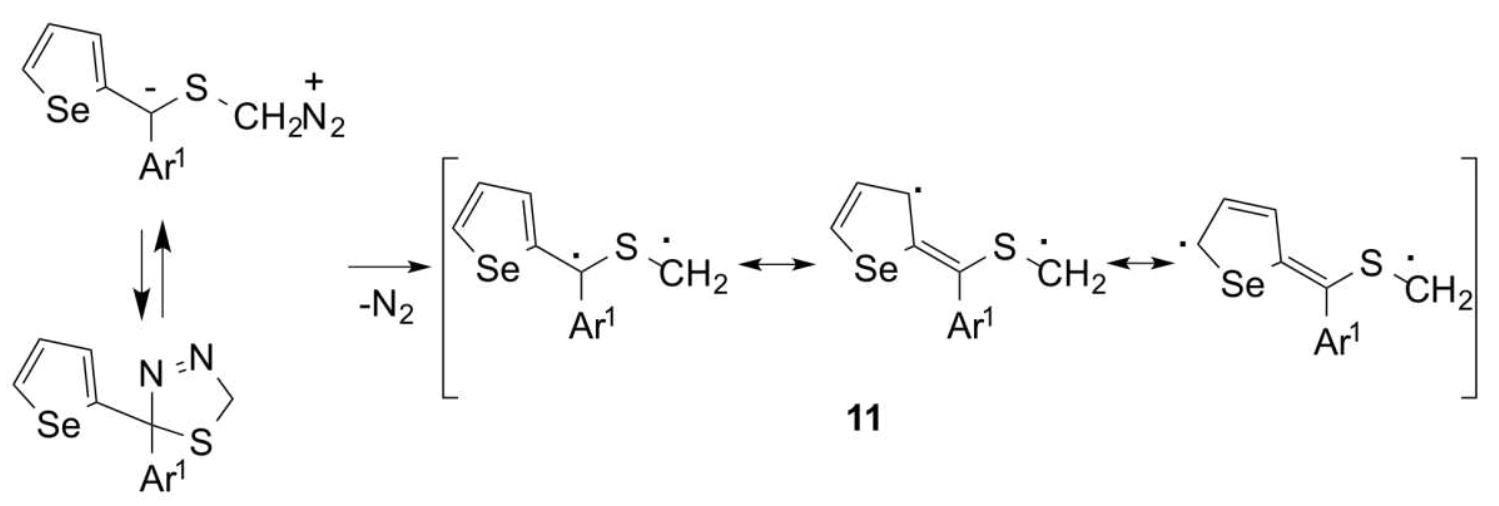


Figure

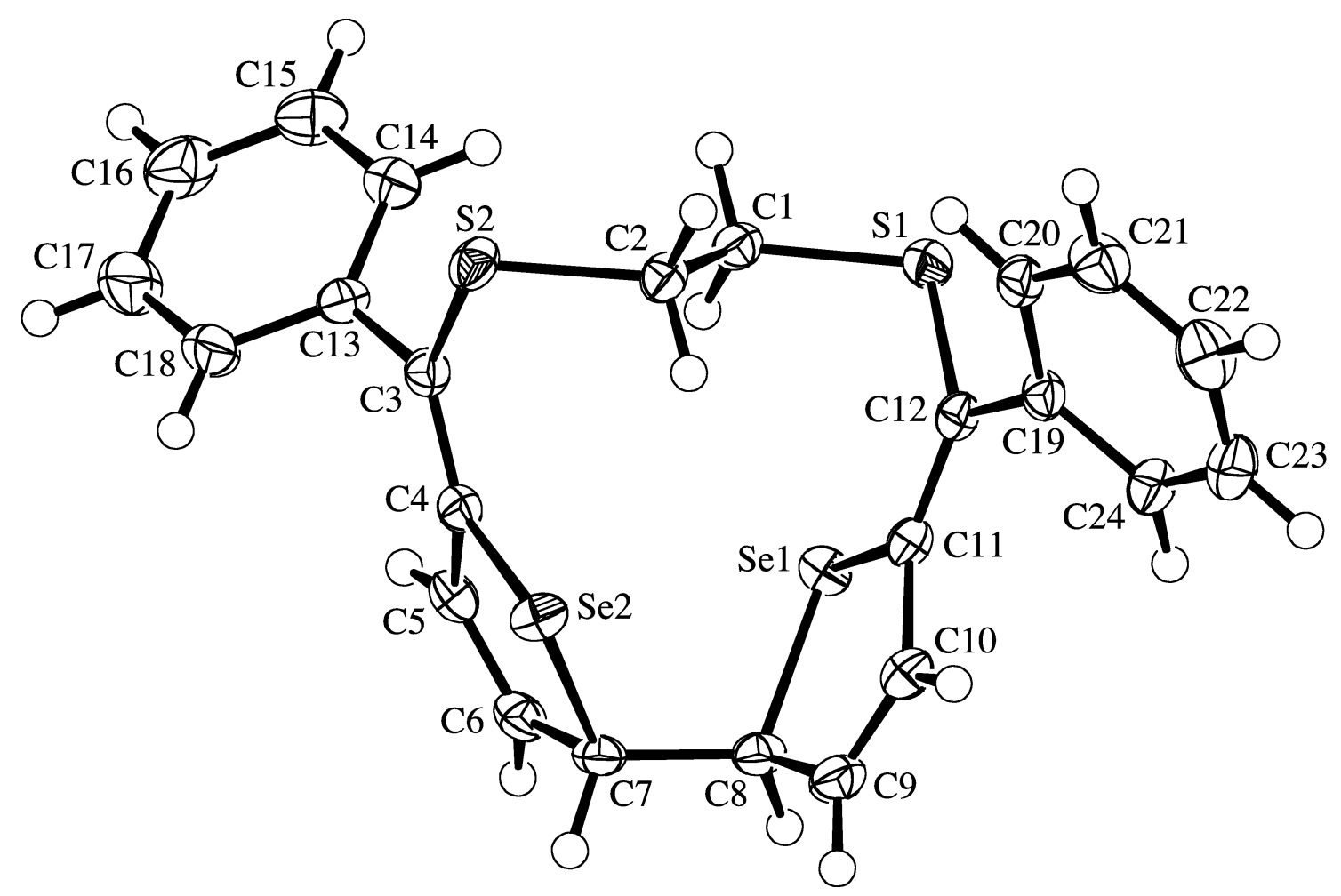




\section{Graphical Abstract}

$\underset{\mathrm{Se}}{\stackrel{\mathrm{N}_{2}}{\longrightarrow}}$<smiles>O=[N+]([O-])c1ccc(C(SCCSC(=C2C=CC=[Se]2)c2ccc([N+](=O)[O-])cc2)=C2C=CC[Se]2)cc1</smiles> 